\title{
DITERPENOS, TRITERPENOS E ESTERÓIDES DAS FLORES DE Wedelia paludosa
}

Geizi Jane A. de Carvalho, Mário Geraldo de Carvalho

Departamento de Química, Instituto de Ciências Exatas, Universidade Federal Rural do Rio de Janeiro, 23851-970 Seropédica - RJ Dalva T. Ferreira, Terezinha de J. Faria

Departamento de Química, Universidade Estadual de Londrina, CP 6001, 86051-970 Londrina - PR

Raimundo Braz-Filho

Setor de Química de Produtos Naturais, LCQUI-CCT, Universidade Estadual do Norte Fluminense, 28015-620 Campos - RJ

Recebido em 4/11/99; aceito em 19/9/00

DITERPENES, TRITERPENES AND STEROIDS FROM FLOWERS OF WEDELIA PALUDOSA. Phytochemical investigation of the flowers of Wedelia paludosa afforded two diterpenes, ent-kaur-16-en19-oic acid and ent-kaur-9(11),16-dien-19-oic acid, three acylated triterpenes, 3ß-O-hexa-decanoylolean-

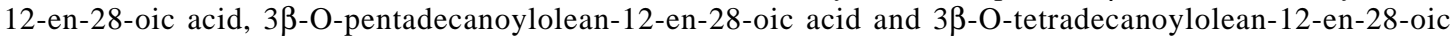

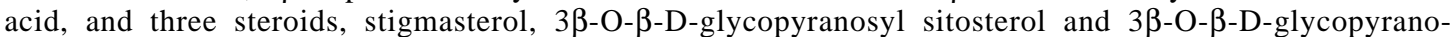
sylstigmasterol. The substances were identified by their spectral data.

Keywords: Wedelia paludosa; kaurenoic acids; 3ß-O-acyloleanoic acids.

\section{INTRODUÇÃOO}

A família Asteraceae compreende cerca de 25.000 espécies distribuídas em aproximadamente 1.100 gêneros, sendo representada no Brasil por cerca 180 gêneros ${ }^{1}$. Esta família aparece com um número relativamente grande de citações bibliográficas, envolvendo farmacognosia, fitoquímica e plantas medicinais ${ }^{2}$.

As atividades farmacológicas reveladas pelos extratos ou substâncias isoladas de espécies do gênero Wedelia despertaram o interesse para estudo químico ${ }^{3}$.

A espécie Wedelia paludosa D. C., usada na medicina popular como expectorante e anticonvulsivo, foi estudada anteriormente após a verificação de atividade antibiótica revelada por testes preliminares envolvendo um extrato bruto das partes aéreas. $\mathrm{O}$ estudo químico desse extrato revelou a presença de sete ácidos caurenóicos, um labdano e uma seco-caurenolactona ${ }^{4}$. Recentemente, foi observada também atividade inseticida em ensaios com esta espécie e uma reinvestigação fitoquímica conduziu ao isolamento e caracterização de duas novas lactonas sesquiterpênicas ${ }^{5}$.

Este artigo descreve os resultados obtidos do estudo químico das flores de um espécimen de Wedelia paludosa. Identificaram-se dois ácidos caurenóicos (1 e 2), estigmasterol (3), glicosídios de estigmasterol (5) e sitosterol (6) e três ésteres derivados do ácido oleanóico (4a-4c). As substâncias 2, 4, 5 e 6 estão sendo registradas pela primeira vez nesta espécie. Os dados de $\mathrm{RMN}{ }^{13} \mathrm{C}$ de 2 estão sendo registrados pela primeira vez na literatura.

\section{RESULTADOS E DISCUSSÃO}

O fracionamento cromatográfico do extrato etéreo das flores de Wedelia paludosa e a análise espectrométrica das frações permitiu a identificação de dois ácidos caurânicos (1 e 2), estigmasterol (3), uma mistura de 3 $\beta$-O- $\beta$-D-glicopiranosilsitosterol (5) e 3 $\beta$-O- $\beta$-D-glicopiranosilestigmasterol (6) e uma mistura de três ésteres $3 \beta-O$-aciloleanóicos $(\mathbf{4 a}, \mathbf{4 b}$ e $\mathbf{4 c})$. Estas substâncias natutais foram identificadas através da análise dos dados espectrais de RMN e massas das frações contendo as substâncias naturais, dos derivados metilados (1a e 2a) e acetilados (5a e 6a) e comparação com dados registrados na literatura.

A análise comparativa dos espectros de RMN ${ }^{13} \mathrm{C}$-HBBD (Hydrogen Broad Band Decoupled) e RMN ${ }^{13} \mathrm{C}$-DEPT (Distortionless Enhancement by Polarization Transfer) de 1 permitiu propor a fórmula parcial $\mathrm{C}_{5}(\mathrm{CH})_{3}\left(\mathrm{CH}_{2}\right)_{10}\left(\mathrm{CH}_{3}\right)_{2}$. Os grupos ácido e vinila foram caracterizados pelos valores dos $\delta_{\mathrm{C}} 185,0\left(\mathrm{CO}_{2} \mathrm{H}\right), 155,5(=\mathrm{C})$ e $103,0\left(=\mathrm{CH}_{2}\right)$ e pelos sinais de dois hidrogênios metilênicos de carbono $\mathrm{sp}^{2}$ em $\delta_{\mathrm{H}} 4,74$ (sl) e 4,79 (sl) presentes no espectro de RMN de ${ }^{1} \mathrm{H}$. Estas informações em conjunto e o pico em $\mathrm{m} / z 302 \mathrm{D}\left([\mathrm{M}]^{++}\right)$revelado pelo espectro de massas e comparação dos deslocamentos químicos dos átomos de carbono $\left(\delta_{\mathrm{C}}\right)$ e hidrogênio $\left(\delta_{\mathrm{H}}\right)$ com valores descritos na literatura ${ }^{6,7}$ permitiram a identificação desta substância natural como ácido ent-caur-16-en-19-óico (1). Este diterpeno (1) foi isolado anteriormente desta espécie4.

A substância 2 foi reconhecida numa mistura com 1. A análise do espectro de RMN ${ }^{1} \mathrm{H}$ da mistura de $\mathbf{1}$ e 2 revelou, além dos sinais do terpeno $\mathbf{1}$, um sinal adicional na região de absorção de hidrogênios olefínicos $\left(\delta_{\mathrm{H}} 5,25, \mathrm{t}, \mathrm{H}-11\right)$, com integração correspondendo a aproximadamente $50 \%$ do valor da intensidade do singleto largo em $\delta_{\mathrm{H}} 4,79(\mathrm{H}-17)$ de 1 . A análise comparativa dos espectros de $\mathrm{RMN}{ }^{13} \mathrm{C}$ (HBBD e DEPT, $\theta=135^{\circ}$ e $\theta=90^{\circ}$ ) permitiu reconhecer, além dos sinais dos carbonos de $\mathbf{1}$, os sinais de uma ligação dupla trisubstituida $\left[\delta_{\mathrm{C}} 138,0(\mathrm{C}-9)\right.$ e $114,8(\mathrm{CH}-11)$ e um sinal de grupo metila adicional $\left(\delta_{\mathrm{C}} 23,8, \mathrm{CH}_{3}-20\right)$.

$\mathrm{O}$ espectro de massas da mistura de $\mathbf{1}$ e $\mathbf{2}$ apresentou picos em $\mathrm{m} / z 302$ (100) correspondente ao íon molecular de $\mathbf{1}$ e em $\mathrm{m} / z 300$ (10) compatível com a presença do diterpeno 2 com estrutura semelhante a $\mathbf{1}$ contendo uma ligação dupla adicional.

A localização da ligação dupla trisubstituida entre os átomos de carbono C-9 e CH-11 foi confirmada pelo resultado de NOE $(2 \%)$ observado no sinal do hidrogênio H-11 $\left(\delta_{\mathrm{H}} 5,25\right)$ com a irradiação na frequência dos hidrogênios metílicos $3 \mathrm{H}$ 20. Estas informações e a comparação dos deslocamentos químicos dos carbonos de $\mathbf{2}$ com valores de modelos relatados na litratura ${ }^{7}$ foram usados para deduzir a estrutura do ácido entcaur-9(11),16-dien-19-oico (2) e atribuir pela primeira vez os deslocamentos químicos dos carbonos deste diterpeno. Esta substância foi também isolada de Wedelia hispida, W. callycina $^{8}, W$. hookenriana ${ }^{9}$ e W. buphthalmiflora ${ }^{2}$.

As estruturas dos esteróides $\mathbf{3}, \mathbf{5}$ e $\mathbf{6}$ foram deduzidas através da análise dos espectros de RMN ${ }^{1} \mathrm{H} \mathrm{e}{ }^{13} \mathrm{C}$ (HBBD e DEPT) de $\mathbf{3}$ e dos derivados acetilados $\mathbf{5} \mathbf{a}$ e $\mathbf{6 a}$, envolvendo a comparação com valores divulgados na literatura para $3^{10,11}, \mathbf{5 a}$ e $\mathbf{6 a}^{11,12}$.

A presença da unidade triterpênica pentacíclica em $\mathbf{4}$ foi reconhecida pelos deslocamentos químicos e multiplicidade dos 
sinais de RMN ${ }^{13} \mathrm{C}$ (HBBD e DEPT), comparação com os valores do ácido oleanóico registrados na literatura ${ }^{13}$ e pelos deslocamentos químicos dos sinais dos hidrogênios $\mathrm{H}-12\left(\delta_{\mathrm{H}}\right.$ $5,25, \mathrm{t}, \mathrm{J}=3,6 \mathrm{~Hz}), \mathrm{H}-3\left(\delta_{\mathrm{H}} 4,50, \mathrm{t}, \mathrm{J}=8,8 \mathrm{~Hz}\right)$ e H-18 $\left(\delta_{\mathrm{H}} 2,80\right.$, dd, J=9,6 e $6,8 \mathrm{~Hz}$ ) presentes no espectro de RMN de ${ }^{1} \mathrm{H}$. Os picos em $\mathrm{m} / z 439(55,4 \mathbf{d}), 248(48,4 \mathbf{4}), 203(48,4 \mathbf{f})$ e 191 $(100, \mathbf{4 g})$ observados no espectro de massas de $\mathbf{4}$ revelaram-se em acordo com a presença desta unidade. Os valores dos deslocamentos químicos do $\mathrm{H}-3\left(\delta_{\mathrm{H}} 4,50, \mathrm{t}, \mathrm{J}=8,8 \mathrm{~Hz}\right)$ e $\mathrm{CH}-3\left(\delta_{\mathrm{C}}\right.$ $80,5)$ permitiram postular a presença de uma função ester no átomo de carbono $\mathrm{CH}-3$.

Os sinais adicionais presentes nos espectros de RMN ${ }^{13} \mathrm{C}$ e RMN ${ }^{1} \mathrm{H}$ foram correlacionados com unidade acila: $\delta_{\mathrm{C}} 173,7$ (C-1'), 34,8 $\left(\mathrm{CH}_{2}-2^{\prime}\right), 31,9\left[\mathrm{CH}_{2}-(\mathrm{n}-3)\right], 29,1\left[\left(\mathrm{CH}_{2}\right)_{\mathrm{n}}\right], 22,5$ $\left[\mathrm{CH}_{2}-(\mathrm{n}-2)\right.$ e $14,1\left(\mathrm{CH}_{3}-\mathrm{n}\right) ; \delta_{\mathrm{H}} 2,30\left(\mathrm{t}, \mathrm{J}=6,0 \mathrm{~Hz}, \mathrm{H}-2{ }^{\prime}\right), 1,24$ $\left[\mathrm{m},\left(\mathrm{CH}_{2}\right)_{\mathrm{n}}\right]$ e $1,00\left(\mathrm{t}, \mathrm{J}=\mathrm{Hz}, \mathrm{CH}_{3}\right)$.

Além dos picos atribuídos aos fragmentos 4d (55), 4e (48), $4 \mathbf{f}(48)$ e $\mathbf{4 g}$ (100), o espectro de massas revelou picos em $\mathrm{m} / \mathrm{z}$ $694(10,4 a), 680(6,4 b)$ e $666(7,4 c)$, que foram correlacionados com os íons moleculares dos três ésteres $(\mathbf{4 a}+\mathbf{4 b}+\mathbf{4} \mathbf{c})$ presentes na mistura. A relação $\mathrm{M}^{++}-498\left[15\left(\mathrm{CH}_{3}\right)+44\right.$
$(\mathrm{O}=\mathrm{C}-\mathrm{O})+439(\mathbf{4 d})]=14 \mathrm{n}$ permitiu deduzir o número de $\mathrm{CH}_{2}$ de cada unidade acila: $4 \mathbf{a}(\mathrm{n}=14), \mathbf{4 b}(\mathrm{n}=13)$ e $\mathbf{4 c}(\mathrm{n}=12)$. Esta dedução foi confirmada pelos picos em $\mathrm{m} / z, 239$ (12), 225 (5) e 211 (8), atribuídos aos íons acilio $\mathbf{4 h}, \mathbf{4 i}$ e $\mathbf{4 j}$, respectivamente. Assim, as estruturas dos ácidos 3 $\beta$-O-hexadecanoilolean-12-en-

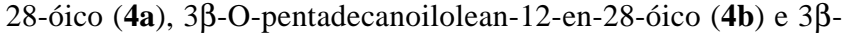
O-tetradecanoilolean-12-en-28-óico $(\mathbf{4 c})$ foram deduzidas. Estes ésteres encontram-se registrados na literatura ${ }^{10,11}$.

\section{PARTE EXPERIMENTAL}

Procedimentos experimentais gerais. Os pontos de fusão (p. f.) foram determinados em placa de Kofler e não foram corrigidos. Os espectros na região de infra-vermelho (IV) foram obtidos em pastilha de $\mathrm{KBr}$ em um espectrômetro PerkinElmer 1420. Os espectros de ressonância magnética nuclear (RMN) foram registrados em um aparelho Brucker AC 200 $\left({ }^{1} \mathrm{H}: 200 \mathrm{MHz} ;{ }^{13} \mathrm{C}: 50.3 \mathrm{MHz}\right)$ e Varian UN $400\left({ }^{1} \mathrm{H}: 400\right.$ $\left.\mathrm{MHz} ;{ }^{13} \mathrm{C}: 100 \mathrm{MHz}\right)$, usando-se $\mathrm{CDCl}_{3}$ como solvente e TMS como referência interna. Os espectros de massas foram obtidos por impacto de elétrons em espectrômetro VG QUATTRO.

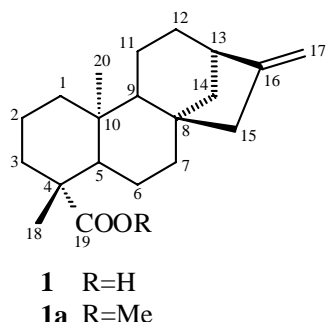

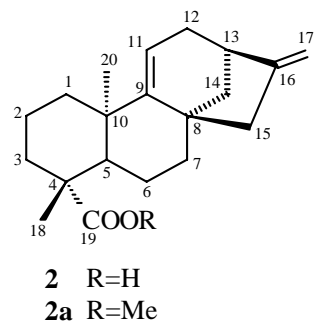<smiles>[R20]C1CCC2(C)C(=CCC3C2CCC2(C)C3CCC2[C@H](C)C=CC(CC)C(C)C)C1</smiles><smiles>CCC(=O)OC1CCC2(C)C(CC[C@]3(C)C4CCC5(C(=O)O)CCC(C)(C)CC5C4=CCC23)C1(C)C</smiles>

$3 \mathrm{R}=\mathrm{H}, 22,23-$ diidro 5 R=Gli, 22,23-diidro 5a $\mathrm{R}=\mathrm{Gli}(\mathrm{Ac})_{4}, 22,23$-diidro $6 \mathrm{R}=\mathrm{Gli}, \Delta^{22}$ 6a $\mathrm{R}=\mathrm{Gli}(\mathrm{Ac})_{4}, \Delta^{22}$ $\begin{array}{ll}\text { 4a } & n=14 \\ \text { 4b } & n=13 \\ \text { 4c } & n=12\end{array}$

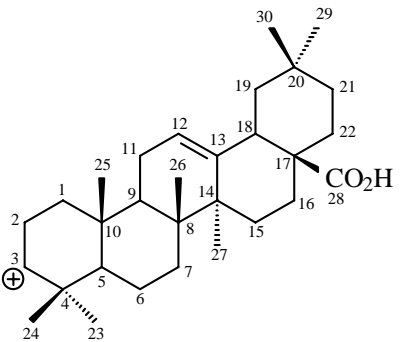

4d $(m / z 439)$<smiles>C=CC1=C(C)CCC2(C(=O)O)CCC(C)(C)CC12</smiles>

4e $(m / z 248)$<smiles>C=CC1=C(C)CCC2CCC(C)(C)CC12</smiles>

4f $(\mathrm{m} / \mathrm{z} 203)$

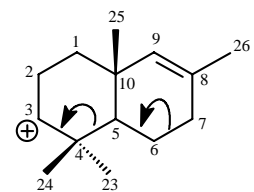

$\mathbf{4 g}(m / z$ 191)<smiles>CCCC#[O+]</smiles>

4h $m / z 239(\mathrm{n}=14), 12 \%$

4i $m / z 225(\mathrm{n}=13), 5 \%$

4j $m / z 211(n=12), 8 \%$ 
Para cromatografia em coluna e camada delgada usou-se sílica gel marca Merck.

Material Vegetal. Um espécime de Wedelia paludosa D.C. foi coletado no mês de outubro de 1990, nas proximidades do Instituto Agronômico do Paraná (IAPAR), Londrina, Paraná, e identificado pela Professora Dra. Ana Odete de Santos Vieira, Departamento de Biologia Animal e Vegetal da Universidade Estadual de Londrina.

Isolamentos dos constituintes químicos. As flores de Wedeila paludosa D.C. foram submetidas a secagem em estufa a $60^{\circ} \mathrm{C}$, convertida a pó $(332,7 \mathrm{~g})$ e extraídas à temperatura ambiente com etanol. A destilação do etanol foi feita sob pressão reduzida e forneceu $131 \mathrm{~g}$ de resíduo. Este resíduo foi submetido à processo de partição com $\mathrm{MeOH}, \mathrm{H}_{2} \mathrm{O}$ e éter de petróleo (700 mL MeOH:90 mL $\mathrm{H}_{2} \mathrm{O}: 1000 \mathrm{~mL}$ de éter de petróleo). $\mathrm{O}$ extrato éter de petróleo foi submetido à cromatografia em coluna de sílica gel, utilizando-se como eluente hexano/ $\mathrm{CH}_{2} \mathrm{Cl}_{2} / \mathrm{AcOEt} / \mathrm{MeOH}$ em gradiente de polaridade, fornecendo 221 frações $(250 \mathrm{~mL}$ cada $)$ que foram reunidas em grupos após análise por cromatografia em camada delgada de sílica. O grupo VIII (frações 103-132) forneceu a substância 1 (723 mg, p. f. $\left.181-182^{\circ} \mathrm{C}\right)$ e a mistura das substâncias 1 e 2 (300 mg, p.f. $181-185^{\circ} \mathrm{C}$ ); o grupo IX (frações 133-142) forneceu a substância 3 (193 mg, p. f. 175-178 ${ }^{\circ}$ C); o grupo VII (frações 90102) foi submetido a cromatografia em camada delgada preparativa, obtendo-se uma mistura dos ésteres $\mathbf{4 a}, \mathbf{4 b}$ e $\mathbf{4 c}$ $(120 \mathrm{mg}$ ); e o grupo XVI (frações 208-212) forneceu a mistura das substâncias 5 e $\mathbf{6}(43,5 \mathrm{mg})$.

Ácido caur-16-en-19-óico (1). Cristais brancos, p. f. 181$182^{\circ} \mathrm{C} ;[\alpha]_{\mathrm{D}}-94,8\left(\mathrm{CHCl}_{3}, 2,5\right)$. Os dados de $\mathrm{RMN}{ }^{13} \mathrm{C}(50,3$ $\left.\mathrm{MHz}, \mathrm{CDCl}_{3}\right)$ e $\mathrm{RMN}$ de ${ }^{1} \mathrm{H}\left(200 \mathrm{MHz} \mathrm{CDCl}_{3}\right)$ revelaram-se em acordo com os valores descritos na literatura ${ }^{6,7}$. Esta substância $(100 \mathrm{mg})$ foi submetida a metilação com diazometano em éter etílico (3 $\mathrm{mL})$, fornecendo o derivado metilado 1a (100 mg). Dados de $\mathrm{RMN}{ }^{1} \mathrm{H}\left(200 \mathrm{MHz}, \mathrm{CDCl}_{3}\right)$ revelaram-se semelhantes aos valores de $\mathbf{1}$, contendo sinal adicional do grupo metoxila em $\delta_{\mathrm{H}} 3,56(\mathrm{~s}, \mathrm{OMe})$.

Ácido caur-9(11),16-dien-19-óico (2). A análise dos espectros de RMN e massas da mistura de 1 e $\mathbf{2}$ permitiu reconhecer os dados de $\mathbf{1}$ e deduzir os valores correspondentes a 2. $\delta_{\mathrm{H}}\left(200 \mathrm{MHz}, \mathrm{CDCl}_{3}\right)$ e em acordo com valores relatados na literatura ${ }^{8}$. $\mathrm{RMN}{ }^{13} \mathrm{C} \delta_{\mathrm{C}}\left(50,3 \mathrm{MHz}, \mathrm{CDCl}_{3}\right): 41,0\left(\mathrm{CH}_{2}-\right.$ 1), 19,0 $\left(\mathrm{CH}_{2}-2\right), 37,7\left(\mathrm{CH}_{2}-3\right), 43,9(\mathrm{C}-4), 57,0(\mathrm{CH}-5), 21,8$ $\left(\mathrm{CH}_{2}-6\right), 41,2\left(\mathrm{CH}_{2}-7\right), 44,2(\mathrm{C}-8), 138,0(\mathrm{C}-9), 38,8(\mathrm{C}-10)$, 114,8 (CH-11), 33,1 ( $\left.\mathrm{CH}_{2}-12\right), 43,8(\mathrm{CH}-13), 39,6\left(\mathrm{CH}_{2}-14\right)$, $49,0\left(\mathrm{CH}_{2}-15\right), 156,0(\mathrm{C}-16), 105,5\left(\mathrm{CH}_{2}-17\right), 28,3\left(\mathrm{CH}_{3}-18\right)$ 185,0 (C-19), 23,8 $\left(\mathrm{CH}_{3}-20\right)$. Esta mistura $(50 \mathrm{mg})$ foi metilada com diazometano em éter etílico $(1,5 \mathrm{~mL})$, fornecendo os derivados metilados 1a e 2a. O espectro de $\mathrm{RMN}$ de ${ }^{1} \mathrm{H}\left(200 \mathrm{MHz}, \mathrm{CDCl}_{3}\right)$ revelou-se semelhante ao de $1+2$, contendo os sinais adicionais em $\delta_{\mathrm{H}} 3,65(\mathrm{~s}, \mathrm{OMe})$, e $3,64(\mathrm{~s}, \mathrm{OMe})$.
Estigmasterol (3). Cristais brancos, p. f. $175-178^{\circ}$ C. Os dados de RMN ${ }^{13} \mathrm{C}$ e RMN ${ }^{1} \mathrm{H}$ revelaram-se em acordo com valores relatados na literatura ${ }^{10,11}$.

Mistura dos Ácidos 3 $\beta$-O-hexadecanoilolean-12-en-28-óico

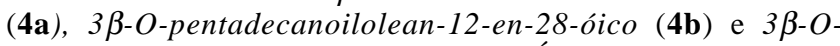
tetradecanoilolean-12-en-28-óico (4c). Óleo amarelo. Estes revelaram-se em acordo com dados descritos na literatura ${ }^{8,9}$.

Tetra-O-acetil-3-O- $\beta$-D-glicopiranosilsitosterol (5a) e tetra$O$-acetil-3-O- $\beta$-D-glicopi-ranosilestigmasterol (6a). A mistura de 5 + 6 (150 mg) foi submetida a acetilação com anidrido acético $(6 \mathrm{~mL})$ e piridina $(6 \mathrm{~mL})$. Após o repouso durante 24 horas à temperatura ambiente e extração do produto acetilado com $\mathrm{CHCl}_{3}$ obteve-se a mistura dos derivados 5a e 6a. Os dados espectrais de RMN ${ }^{13} \mathrm{C}$ e $\mathrm{RMN}{ }^{1} \mathrm{H}$ revelaram-se semelhantes aos valores relatados na literatura ${ }^{9,12}$.

\section{AGRADECIMENTOS}

Os autores agradecem ao CNPq, CAPES e FAPERJ pelos auxílios e bolsas concedidas. Agradecemos também à Professora Ana Odete de Santos Vieira, Departamento de Biologia Animal e Vegetal da Universidade Estadual de Londrina, Londrina, Paraná, Brasil, pela identificação da planta.

\section{REFERÊNCIAS}

1. Barroso, G. M.; Sistemática de Angiosperma do Brasil, 2a , Ed. UFV -MG, 1986.

2. Miles, D. H. ; Chittawong, V.; Payne, A. M.; J. Agric. Food Chem. 1990, 38, 1591.

3. Bruneton, J.; Pharmaconosy, Phytochemistry, Medicinal Plants, $2^{\text {nd }}$ Ed., Lavoisier, N. Y., USA, Intercept Ltd, Adover, England, UK, 1995.

4. Roque, N. F.; Giannella, T. L.; Giesbrecht, A. M.; Barbosa, R. C. C. B. C.; Rev. Latinoam. Quim. 1987, 18, 110.

5. Ferreira, D. T.; Levorato, A. R.; Faria, T. J.; Carvalho, M. G. de; Braz-Filho, R.; Nat. Prod. Lett. 1994, 4, 1.

6. Hasan, C. M.; Healey, T. M.; Waterman, P. G.; Phytochemistry 1982, 21, 1365.

7. Atta-ur-Rahman and Ahmad, V. U., ${ }^{13}$ C NMR of Natural Products, Vol 2, Premium Press, N. Y., 1992.

8. Herz, W.; Kulanthaivel, P.; Phytochemistry 1984, 23, 2271.

9. Bohlmann, F.; Zdero, C.; King, R. M.; Robinson, H.; Phytochemistry 1982, 9, 2329.

10. Kojima, H.; Sato, N.; Hatano, A.; Ogura, H.; Phytochemistry 1990, 29, 2351.

10. Goulart, M. O. F.; Sant'ana; A. E. G.; Lima, R. A.; Cavalcante, S. H.; Carvalho, M. G. de; Braz-Filho, R.; Quim. Nova 1993, 16, 95

11. Akihisa, T.; Ghosh, P.; Thakur. S.; Oshikiri, S.; Tamura, T.; Matsumoto, T.; Phytochemistry 1988, 27, 241.

12. Mahato, S. B.; Kundu, A. P.; Phytochemistry 1994, 37, 1517. 